\title{
Bio-inspired octopus robot based on novel soft fluidic actuator
}

\author{
Jan Fras $^{1}$, Yohan Noh ${ }^{2}$, Mateusz Macias ${ }^{3}$, Helge Wurdemann ${ }^{4}$, and Kaspar Althoefer ${ }^{1}$
}

\begin{abstract}
Many modern roboticists take inspiration from biology to create novel robotic structures, including those that are modeled after the octopus. This paper advances this trend by creating soft robots modeling the complex motion patterns of octopus tentacles employing a bio-mimetic approach. The proposed octopus robot is entirely made from soft material and uses a novel fluidic actuation mechanism that allows the robot to advance forward, change directions and rotate around its primary axis. The paper presents the robot's design and fabrication process. An experimental study is conducted showing the feasibility of the proposed robot and actuation mechanism.
\end{abstract}

\section{INTRODUCTION}

Soft robots are widely considered for different applications in a variety of areas. They operate in a safe way, often outperforming rigid-link robots in this respect [1] and offering a type of motion and deformation that traditional robots can not achieve. Bio-inspiration was often the driving force behind creating novel and innovative soft robots [2][5]. Soft robotics has not only challenged the fields of robot arms and manipulators, but also the area of mobile robots, whereby creating bio-inspired systems that are able to walk, crawl, jump, roll or swim [5]-[8]. One particular area, underwater exploration, can benefit very much from soft robotics solutions, as soft robots are able to easily mimic very complex movements of underwater creatures [9], [10]. In particular, several robots inspired by the octopus have been developed. In [4] an eight arm octopus like robot is presented. It swims employing sculling movements of its arms. In [3] another octopus robot is presented. It is able to mimic octopus crawling locomotion and its arms are made of soft and flexible materials [11]. In both of the above cases, the arms are made of flexible material; however, the rest of the robot is rigid. In both cases, the arms are driven by electrical motors embedded in their bases to achieve locomotion - each arm itself is only a passive extension of a rigid shaft attached to a motor. Involving electric motors in the design leads to an overall implementation that is

*The research has received funding from the European Commission's project Horizon 2020 Research and Innovation Programme, project FourByThree under grant agreement No 637095 .

${ }^{1} \mathrm{~J}$. Fras and K. Althoefer are with the Centre for Advanced Robotics @ Queen Mary (ARQ), Faculty of Science and Engineering, Queen Mary University of London, London E1 4NS, UK j. fras@qmul.ac.uk, k. althoefer@qmul.ac.uk

${ }^{2}$ Y. Noh is with the Department of Biomedical Engineering and the Department of Informatics, King's College London, London WC2R 2 LS, UK yohan. noh@kcl.ac.uk

${ }^{3} \mathrm{M}$. Macias is with Industrial Research Institute for Automation and Measurements, Warsaw, Poland mmacias@piap.pl

${ }^{4} \mathrm{H}$. Wurdemann is with the Department of Mechanical Engineering, UCL, London WC1E 7JE, UK h. wurdemann@ucl.ac.uk to a large extent rigid, diverting from the biological role model considerably. Further, using tendon-based actuation is another aspect where these robots differ significantly from the actuation principles of the octopus.

In this paper we present an entirely soft octopus robot (Figure 1) whose locomotion is achieved using a fluidic actuation principle. Our robot consists of eight bio-inspired arms that actively deform and bend during the actuation cycle. Unlike other octopus-like robots our solution is entirely soft with no rigid elements. The device swims due to the direct deformation of the active parts of its arms, using fluidic actuation chambers. Its arms or tentacles make use of a novel type of soft continuum fluidic actuator that is able to bend in a different range of directions and bending angles as a function of the chamber pressure. Thus the robot can move in a way not achievable for another solutions presented so far, including forward propulsion, turning and rotation around the primary axis.

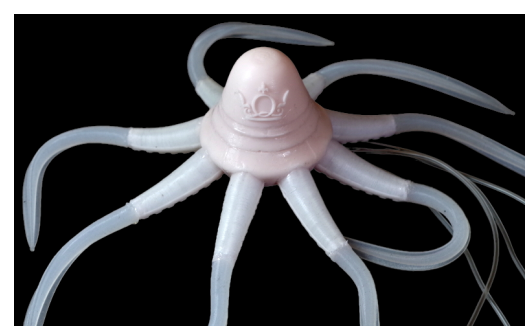

(a)

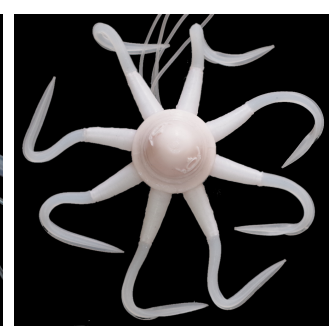

(b)
Fig. 1: The octopus robot. (a) - side view, (b) - top view.

\section{DESIGN}

\section{A. General design}

The various actuation chambers that are the fundamental elements of our octopus robot arms are made of two types of silicone materials, SmoothOn Ecoflex 0030 and SmoothSil 940 [12], [13]. Polyester thread is used to reinforce the outer walls of the chambers. The main body of the robot is entirely made of Smooth-Sil 940. The eight identical arms are made of a combination of Smooth-Sil 940 and Ecoflex 0030. Each arm contains parts that are active (actuation chambers) and those that are passive (extended tails). The passive part of each arm is not actuated and designed to generate the thrust while moving. When actuated the active part of an arm experiences a curling movement; it is noted that this movement alone would not generate any significant force. Hence, the arms are extended by long tails that are pushed through the water amplifying the motion of the active part and achieving forward thrust. 


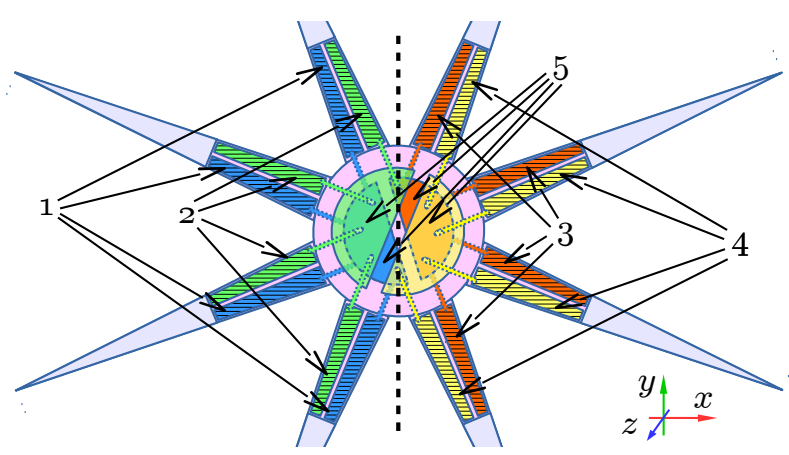

Fig. 2: The robot's design. 1,2,3 and 4 - independent actuator groups, 5 - internal pressure distribution chambers. Body diameter: $55 \mathrm{~mm}$, body height: $53 \mathrm{~mm}$, total diameter with arms straighten: $335 \mathrm{~mm}$.

In our design we assumed both sides of the robot to be actuated independently in order to enable the robot to turn. For that reason four arms are grouped together. As each arm has two degrees of freedom, two groups of arms require four independent pressure signals. The actuation fluid is provided to the robot through flexible pipes and distributed between the arms by internal channels within the robot body. The structure and distribution of the arms is designed to allow the robot to swim forward, to change its heading in all directions, and to rotate around $z$ axis in order to effectively navigate the three dimensional space. The structure and the dimensions of the implemented robot are shown in Figures 2, 4 and 5 . The robot behavior as a function of the pressure in the respective actuator group is shown in Figure 3.

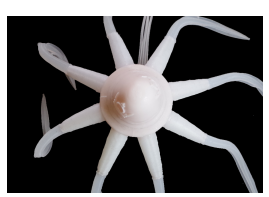

(a)

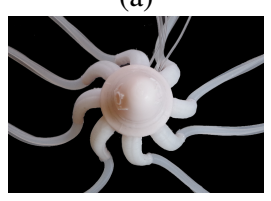

(d)

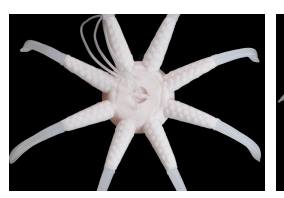

(b)

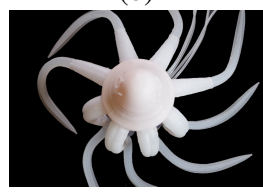

(e)

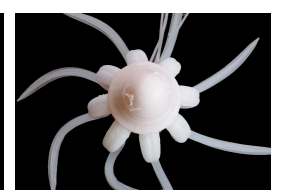

(c)

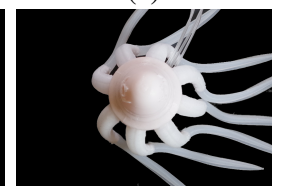

(f)
Fig. 3: Different actuation patterns. (a),(b) - passive robot, top and bottom view, respectively, (c) - same pressure for all actuation chambers, forward movement, (d) - axial activation symmetry $\left(2^{\text {nd }}+4^{\text {th }}\right.$ actuation groups), rotation along $Z$ axis, (e) - one side activation $\left(1^{s t}+2^{\text {nd }}\right.$ actuation groups), rotation around $y$ axis, (f) - two sides activation, $\left(1^{\text {st }}+4^{\text {th }}\right.$ actuation groups), rotation around $x$ axis.

\section{B. The arm}

The actuator construction originates in the fluidic chambers of the soft manipulator developed as part of EU project STIFF-FLOP [14]; inspiration has also been taken from the fingers developed as part of a pneumatic grasper presented

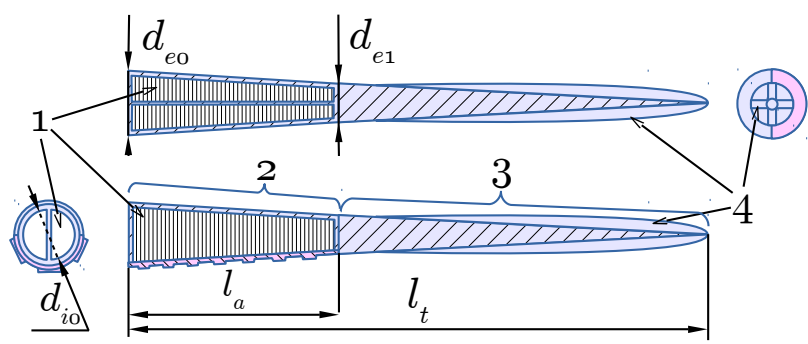

Fig. 4: The robot's arm design. 1 - actuation chambers, 2 and 3 - active and passive parts of the arm, consequently, 4 - finlike surfaces. $l_{a}=40 \mathrm{~mm}, l_{t}=140 \mathrm{~mm}, d_{i 0}=10 \mathrm{~mm}$, $d_{e 0}=15 \mathrm{~mm}, d_{e 1}=9 \mathrm{~mm}$. Blue colour: soft silicone Ecoflex, pink colour: stiff silicone Smooth-Sil.

in [15]. The robot arm presented here has a conical shape and contains active and passive parts, Figure 4. The active part contains two actuation chambers and is composed of several layers of silicone reinforced with polyester thread that forms a helix with a very small pitch around each actuation chamber, Figure 5.

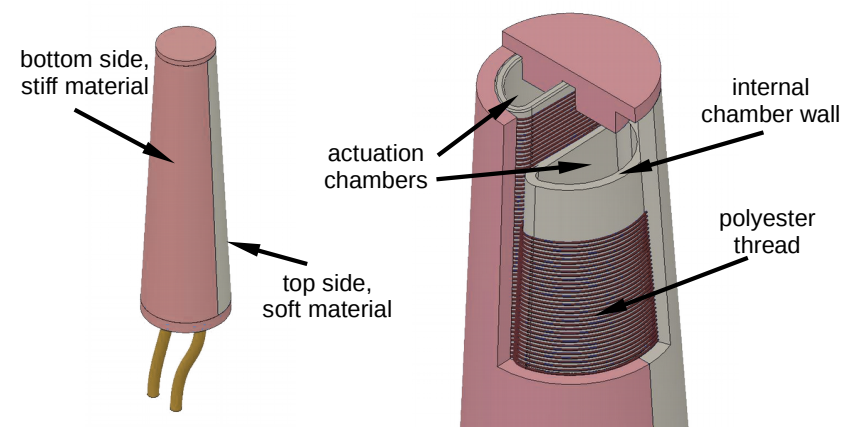

Fig. 5: The active part of the arm - the actuator. Actuation chambers reinforced with the polyester thread shown.

Such a reinforcement allows the structure of the actuator to bend and elongate while, at the same time, reducing radial deformation and thus eliminating the ballooning effect. The bottom side of the actuator is covered with a layer of stiff silicone (Smooth-Sil) while the rest of it is made of the soft one (Ecoflex). For that reason, the pressurized actuator extends more on the top than on the bottom that results in curling motion, see Figure 6. The geometry of the new actuator maximizes the actuation area, as for the bending the whole actuation cross-section is used. At the same time, the new design enables curvature and bending direction to be controlled appropriately varying the pressure values in the two actuation chambers (see Figure 6). The bending motion of the actuator results from different types of elasticity of its layers and its direction can be changed in some range by pressurizing the chamber sections with different pressure values. Previously presented actuators that maximized the actuation chamber cross-section area don't have the ability to change the fixed bending plane [14], [16]-[18]. Other 
actuators that are capable of the bending plane control are usually divided in several actuation chambers and bend due to unsymmetrical actuation. During the actuation only some subset of the chambers is pressurized and thus the actuation volume is limited [14], [19]. In case of the proposed actuator the whole actuation volume (both sides of the actuator) can be pressurized when high efficiency is required, while the bending direction still can be changed by providing different pressures to different actuator sides. This is a novel aspect of our new design, departing from the earlier designs with their single-chamber structure that allowed bending in one plane only, or used only small part of the possible actuation area to enable the bending direction control. More detailed description of the actuator can be found in [20].

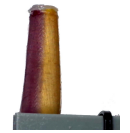

(a)

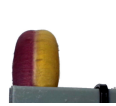

(b)

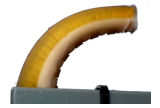

(c)

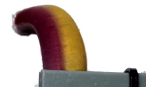

(d)

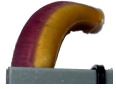

(e)
Fig. 6: The actuator, a) passive, top view, b) and c) symmetrically bent, pressures equal, top view and side view respectively, d) bent to the left, right chamber pressure higher than left, e) bent to the right, left chamber pressure higher than right. Left and right actuation chambers shown in red and yellow consequently.

\section{MANUFACTURING}

The robot is made of silicone material cast in the 3Dprinted molds produced with desktop 3D printer, Zortrax $\mathrm{m} 200$. The process consists of several stages as described below.

\section{A. The arm}

All the arms are produced within a single set of molds in one cycle. The process is very similar to the process described in [15]. It consists of internal cores winding with reinforcement fiber, casting an external top and bottom layers of the actuator (Figures $7 \mathrm{a}$ and $7 \mathrm{~b}$ ), inserting the preprepared semi-cylindrical internal chambers (2 chambers per arm), sealing the tip of the actuator with stiff silicone (Figure 7c) and attaching the passive tail extension.

\section{B. The robot body}

The main part of the body is cast in the dedicated mold as seen in Figure $7 d$. The main part of the body contains 8 sockets for the arms with 2 pressure channels per arm, see Figures $8 \mathrm{a}$ and $8 \mathrm{~b}$. The arms are connected to the body using the same material the body is made of (Smooth-Sil 940).

The body consists of a several empty chambers used as arms connection. All those chambers are open after the first casting step. The mold used for that procedure is shown in Figure $7 \mathrm{~d}$. After the silicone is cured, the arms are connected and all connections are checked. If everything is sealed properly the top and bottom chambers of the body are closed. This is the last step and completes the fabrication procedure.

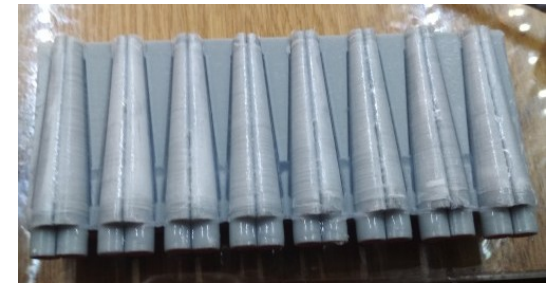

(a)

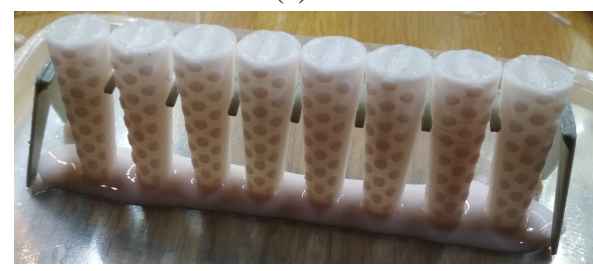

(c)

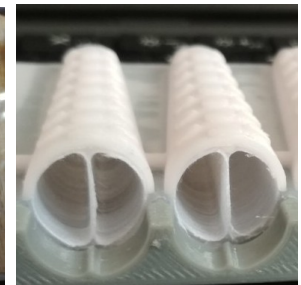

(b)

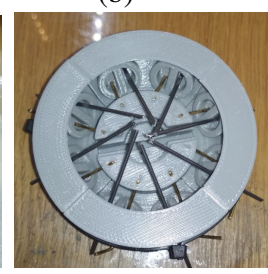

(d)
Fig. 7: Fabrication steps: (a) - top actuator layer cast, (b) arms internal chambers inserted, (c) - tips of the chambers being sealed, (d) - mold for the octopus body.

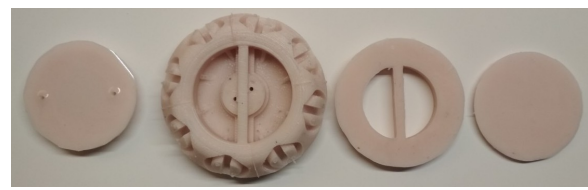

(a)

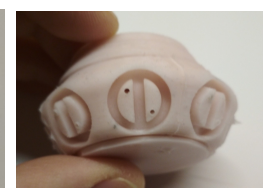

(b)
Fig. 8: The robot's body. a) Parts of the body, internal distribution chambers visible (compare fig. 2). b) body assembled, arm socket with two separate pressure inlets presented.

The assembled robot is presented in Figure 1. The weight of the finished robot is approximately $120 \mathrm{~g}$.

\section{TESTS}

The robot capabilities in terms of velocity and generated force as a function of input pressures has been tested and are discussed below. The final target environment for the robot is water, however, for the tests air actuation was used. This is due to the high water viscosity that generates resistance and reduce the actuation medium flow resulting in very slow robot actuation. Air actuation, however, causes the actuators to change their volume significantly while their weight changes slightly. The additional air volume in robot body increases the buoyancy and allows it to swim out of the water. For that reason the velocity tests were performed on the surface. For the force tests the robot was fixed to a lever that kept it under the water surface despite the varying buoyancy.

\section{A. Test setup}

All the experiments were conducted in a water tank made of transparent acrylic glass. The robot was powered by pressurized air supplied with $1 \mathrm{~m}$ long and $2 \mathrm{~mm}$ wide flexible tubes. The pressure was regulated at the tube inlet with a Camozzi k8p pressure regulator. For all the actuation trials only one tube was used to provide the pressure to the 


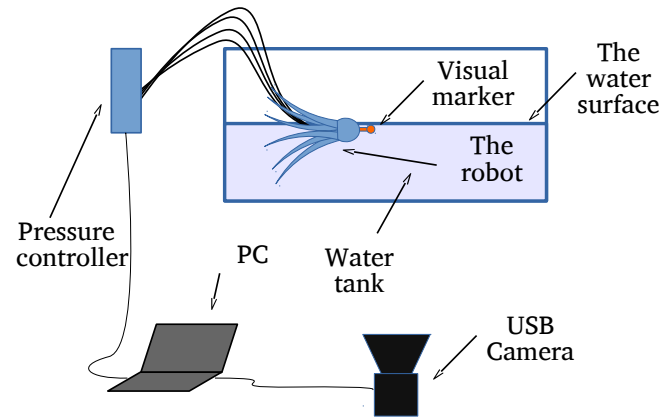

(a)

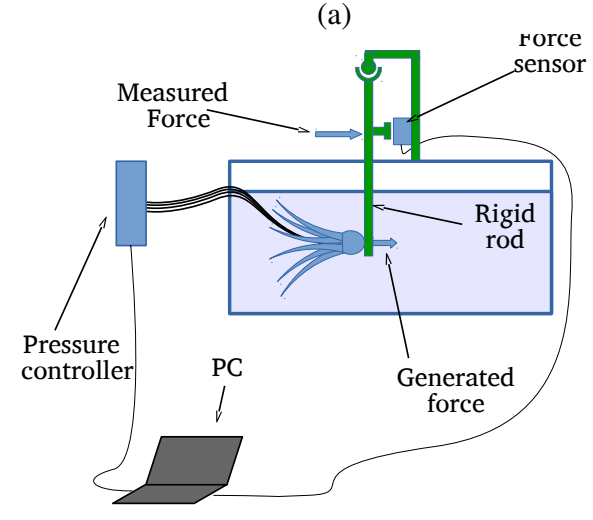

(b)

Fig. 9: Test stands. a - configuration for velocity test, bconfiguration for force test.

robot. Using a small connector the pressurized air was then distributed to relevant distribution chambers to activate the desired actuator groups (groups $1-4$ for velocity and force tests, 1 and 3 for twist motion, 1 and 2 for turning motion).

\section{B. Velocity test}

For the velocity measurements the robot was allowed to freely move inside the tank and powered with a sequence of pressure pulses of a given frequency and a given pressure value. While progressing through the tank, the octopus robot was observed with a camera; to simplify motion tracking, the robot had a colored marker attached, Figure 9a. Using image analysis techniques, the robot position was extracted from the recorded image sequence and combined with pressure data. The results are presented in Figure 11. The data presents three full actuation cycles and is an average with error bars from six trials. The robot replicates the arm swimming pattern of the octopus [21]. A sequence of frames extracted from the recorded movie is presented in Figure 10.

\section{Force test}

The robot has been tested in terms of the forces it can generate during actuation. The setup used for that procedure is presented in Figure 9b. During the test, the robot was attached to the bottom end of a rod suspended on a hinge. The rod was aligned vertically in a way that only the robot was submerged. In one third of the rod length a force sensor was attached. During the actuation the robot was pressing the rod that in turn was pressing the sensor, see Figure $9 \mathrm{~b}$. For the experiment a single axis force sensor has been used [22][24]. During the actuation the robot was exerting a force on a rod that was transferred to the sensor without any movement. Knowing the sensor position and the length of the rod, the measured force was recalculated using the law of the lever.

The recorded forces are presented in Figure 12

\section{Manoeuvres capabilities evaluation}

The turning and twisting manoeuvres have not yet been quantified, but the initial test shown that the robot is capable of the assumed motion types, see Figure 14 and Figure 13.

\section{RESUlTS AND Discussion}

The initial test shown that the robot is capable of the assumed motion; it can proceed, twist and turn. For the tested actuation pattern that is a sequence of pressure pulses of 1 bar value, $1.5 \mathrm{~s}$ period and $0.3 \mathrm{~s}$ pulse time, the average velocity of the presented robot is approximately $3.8 \mathrm{~cm} / \mathrm{s}$ and around $9 \mathrm{~cm} / \mathrm{s}$ at pick. This pattern was chosen to show current design capability. Pressure was set on safe level and was removed when a significant velocity was achieved. Next pulse was applied when velocity dropped near zero The thrust generated by the robot actuated in the same manner but in a fixed position (no velocity in relation to the water) in average is approximately $0.011 \mathrm{~N}$ and around $0.11 \mathrm{~N}$ at the pick value. The generated force has a low value but sufficient to drive the robot. The rotational and twist motion have not yet been quantified.

As the robot is powered by an external pressure controlled by a regulator connected to the robot with a relatively thin and long pipe, its value in the actuators varies from the requested one. This is due to the regulators maximum flow (which is limited), the air viscosity and compressibility. Thus the actual actuation pressure is far smaller than the requested one. The measurements of the pressure near to the regulator outlet and near to the robot inlet are presented in Figure 15. Such a disparity has to be taken into account when designing a proper robot control algorithms and is a significant drawback of an external power source.

\section{CONCLUSIONS AND FUTURE WORK}

In this paper we presented a soft, bio-inspired mobile octopus robot. The robot is based on a novel type of actuator that is able to control the bending direction and use the whole actuation area at the same time. In this paper we provide our design strategy and describe the chosen manufacturing process. An experimental study was conducting showing the feasibility of the new actuation mode for octopus like robots, capable of life-like motion patters moving forward, turning and rotating around the main axis.

At the current stage, the power source, used in this initial study, is an external system providing pressurized air - this proved a useful way to show feasibility but limits the realworld applicability. It is also noted that the length of the 


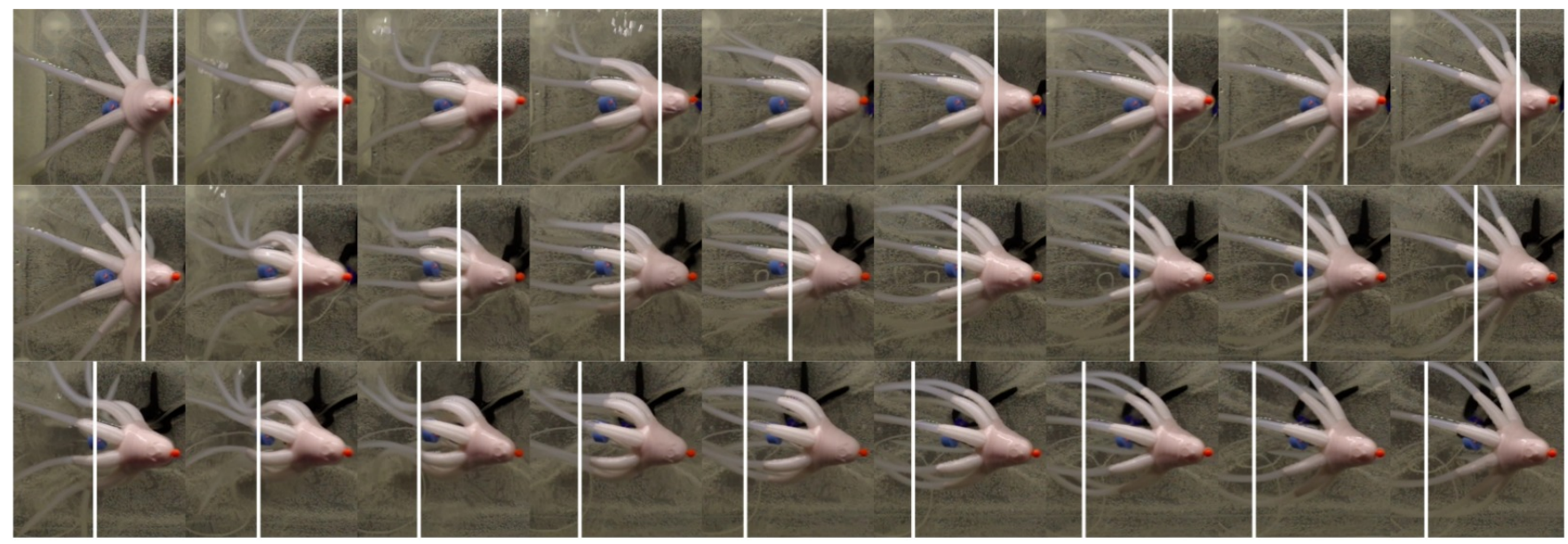

Fig. 10: Movement of the robot; forward motion, three cycles presented. Video recorded at $30 \mathrm{fps}$. Every fifth frame presented.

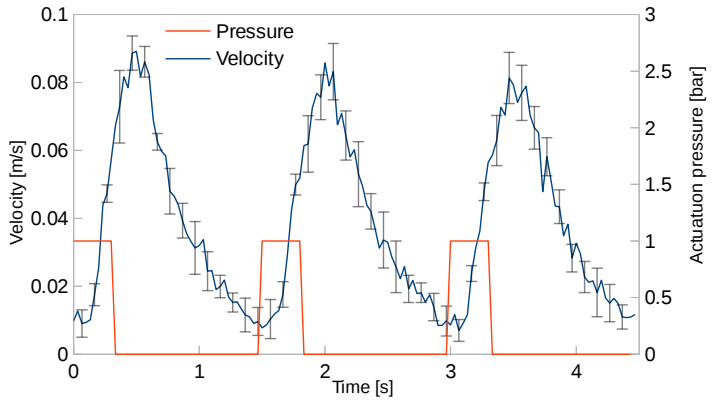

Fig. 11: Velocity test result. The plot presents average velocity values of 6 trials with error bars. Three full actuation cycles are presented.

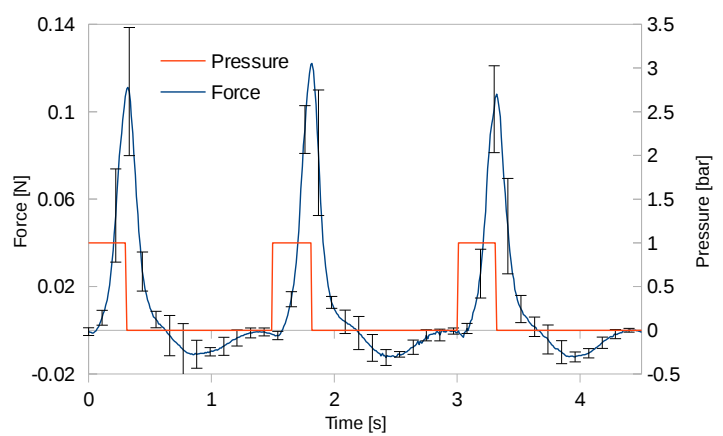

Fig. 12: Force test result. The plot presents the average forces recorded across 8 trials with error bars. Three full actuation cycles are presented.

air-feeding tubes introduces issues including delays of the pressure change due to the actuation medium's viscosity. The medium we used for the experiments here was air; however, the medium we aim to use in the final system is a liquid like water or oil. Such a solution would allow controlling the volume instead of the pressure; this would be achieved much more easily and would not affect the robot's displacement.

The structure of the presented robot results from the authors experience and intuition and have shown to work as desired. It is, however, suboptimal and can be improved in terms of the arms effectiveness, mass distribution and flow optimization. To achieve an optimized robot with regards to its motion characteristics, we intend to explore detailed modeling based on finite element analysis.

Further steps the authors consider as future work is to create miniaturized and mobile power source solutions that could be embed in the robot, and thus increase its application capabilities.

\section{REFERENCES}

[1] A. Stilli, L. Grattarola, H. Feldmann, H. A. Wurdemann, and K. Althoefer. Variable stiffness link (vsl): Toward inherently safe robotic manipulators. In 2017 IEEE International Conference on Robotics and Automation (ICRA), pages 4971-4976, May 2017.

[2] A. Stilli, H. A Wurdemann, and K. Althoefer. Shrinkable, stiffnesscontrollable soft manipulator based on a bio-inspired antagonistic actuation principle. In International Conference on Intelligent Robots and Systems, pages 2476-2481. IEEE, 2014.

[3] M Cianchetti, M Calisti, L Margheri, M Kuba, and C Laschi. Bioinspired locomotion and grasping in water: the soft eight-arm octopus robot. Bioinspiration and Biomimetics, 10(3):035003, 2015.

[4] M. Sfakiotakis, A. Kazakidi, N. Pateromichelakis, and D. P. Tsakiris Octopus-inspired eight-arm robotic swimming by sculling movements. In 2013 IEEE International Conference on Robotics and Automation, pages 5155-5161, May 2013.

[5] Alireza Ramezani, Soon-Jo Chung, and Seth Hutchinson. A biomimetic robotic platform to study flight specializations of bats. Science Robotics, 2(3), 2017

[6] Robert F. Shepherd, Filip Ilievski, Wonjae Choi, et al. Multigait soft robot. Proceedings of the National Academy of Sciences, 108(51):20400-20403, 2011.

[7] Koichi Suzumori, Satoshi Endo, Takefumi Kanda, Naomi Kato, and Hiroyoshi Suzuki. A bending pneumatic rubber actuator realizing softbodied manta swimming robot. In Robotics and Automation, 2007 IEEE International Conference on, pages 4975-4980. IEEE, 2007.

[8] M. Calisti, G. Picardi, and C. Laschi. Fundamentals of soft robot locomotion. Journal of The Royal Society Interface, 14(130), 2017.

[9] Yong Zhong and Ruxu Du. Design and implementation of a novel robot fish with active and compliant propulsion mechanism. In Robotics: Science and Systems, 2016.

[10] Andrew D Marchese, Cagdas D Onal, and Daniela Rus. Autonomous soft robotic fish capable of escape maneuvers using fluidic elastomer actuators. Soft Robotics, 1(1):75-87, 2014.

[11] M Cianchetti, A Arienti, M Follador, B Mazzolai, P Dario, and $\mathrm{C}$ Laschi. Design concept and validation of a robotic arm inspired by the octopus. Materials Science and Engineering: C, 31(6):12301239,2011

[12] Ecoflex 00-30 product page. 


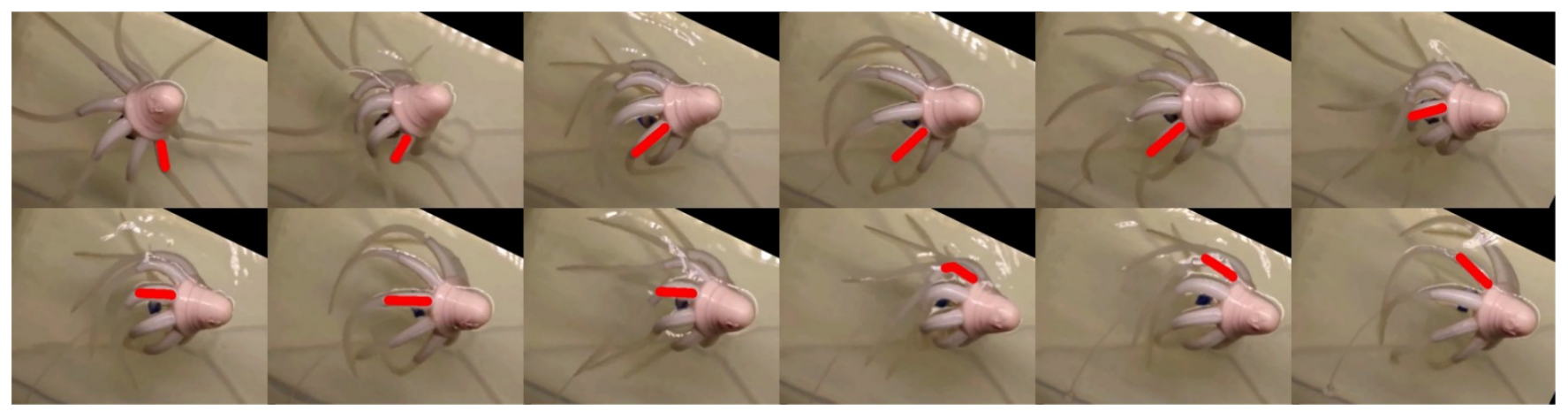

Fig. 13: Movement of the robot; twisting motion. Two actuation cycles presented. For better clarity one of the arms coloured with red.

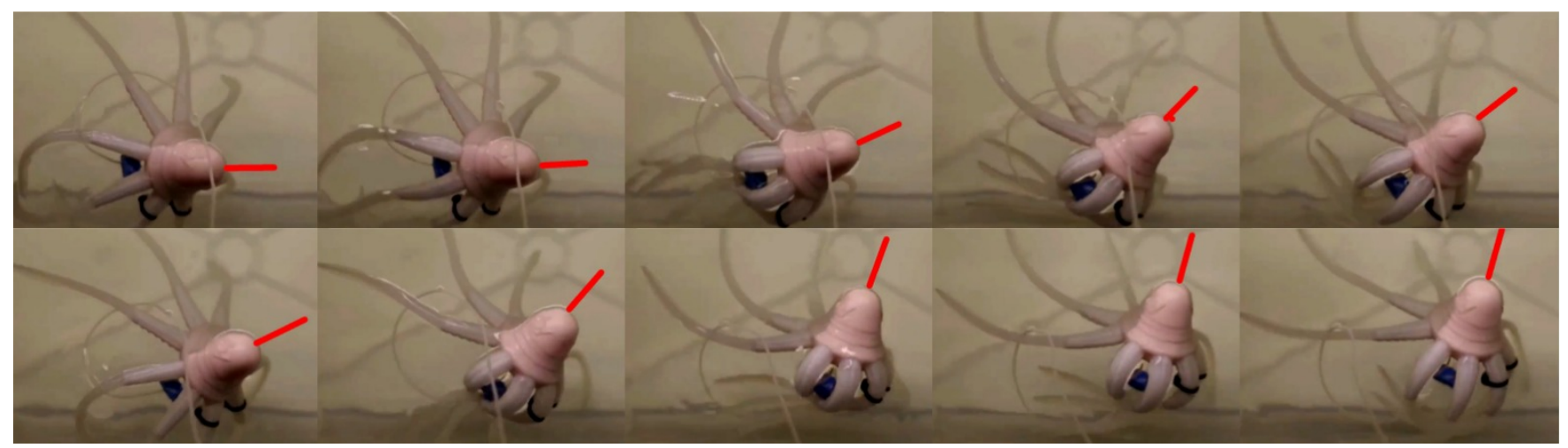

Fig. 14: Movement of the robot; turning motion. Two cycles presented. For better clarity the direction of the robot marked in red.

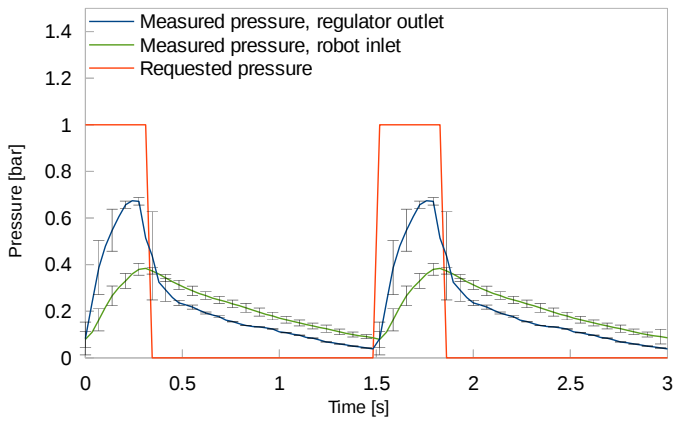

Fig. 15: The disparity between requested and actual pressures in the system.

[13] Smooth-Sil 940 product page.

[14] J. Fras, J. Czarnowski, M. Macias, J. Glowka, M. Cianchetti, and A. Menciassi. New stiff-flop module construction idea for improved actuation and sensing. In International Conference on Robotics and Automation, pages 2901-2906. IEEE, 2015.

[15] J. Fras, M. Macias, F. Czubaczynski, P. Salek, and J. Glowka. Soft flexible gripper design, characterization and application. In International Conference SCIT, Warsaw, Poland. Springer, 2016.

[16] Fionnuala Connolly, Panagiotis Polygerinos, Conor J. Walsh, and Katia Bertoldi. Mechanical programming of soft actuators by varying fiber angle. Soft Robotics, 2(1):26-32, March 20152015.

[17] Raphael Deimel and Oliver Brock. A novel type of compliant and underactuated robotic hand for dexterous grasping. The International Journal of Robotics Research, 35(1-3):161-185, 2016.
[18] J. Fras, Y. Noh, H Wurdemann, and K. Althoefer. Soft fluidic rotary actuator with improved actuation properties. In International Conference on Intelligent Robots and Systems. IEEE, 2017.

[19] K. Suzumori, S. Iikura, and H. Tanaka. Development of flexible microactuator and its applications to robotic mechanisms. In International Conference on Robotics and Automation, pages 1622-1627. IEEE, 1991.

[20] J. Fras, Macias M., Y. Noh, and K. Althoefer. Fluidical bending actuator designed for soft octopus robot tentacle. In submitted to International Conference on Soft Robotics (RoboSoft). IEEE, 2018.

[21] Asimina Kazakidi, Michael Kuba, Alex Botvinnik, Michael Sfakiotakis, Tamar Gutnick, Shlomi Hanassy, Guy Levy, John A Ekaterinaris, Tamar Flash, Binyamin Hochner, et al. Swimming patterns of the octopus vulgaris. In 22nd Annual Meeting NCM Society, pages 2329, 2012.

[22] Y. Noh, Akihiro Shimomura, Masanao Segawa, Hiroyuki Ishii, J. Solis, Atsuo Takanishi, and Kazuyuki Hatake. Development of tension/compression detection sensor system designed to acquire quantitative force information while training the airway management task. In 2009 IEEE/ASME International Conference on Advanced Intelligent Mechatronics, pages 1264-1269, July 2009.

[23] Y. Noh, M. Segawa, A. Shimomura, H. Ishii, J. Solis, A. Takanishi, and K. Hatake. Development of the airway management training system wka-2 designed to reproduce different cases of difficult airway. In 2009 IEEE International Conference on Robotics and Automation, pages 3833-3838, May 2009.

[24] Y. Noh, K. Ebihara, M. Segawa, K. Sato, C. Wang, H. Ishii, J. Solis, A. Takanishi, K. Hatake, and S. Shoji. Development of the airway management training system wka-4: For improved high-fidelity reproduction of real patient conditions, and improved tongue and mandible mechanisms. In 2011 IEEE International Conference on Robotics and Automation, pages 1726-1731, May 2011. 